\title{
Research on Improved Adaptive Control for Static Synchronous Compensator in Power System
}

\author{
Chao Zhang, ${ }^{1}$ Aimin Zhang, ${ }^{2}$ Hang Zhang, ${ }^{1}$ Yingsan Geng, ${ }^{1}$ and Yunfei Bai ${ }^{1}$ \\ ${ }^{1}$ State Key Laboratory of Electrical Insulation and Power Equipment, Xian Jiaotong University, Xian, Shaanxi 710049, China \\ ${ }^{2}$ School of Electronic and Information Engineering, Xian Jiaotong University, Xian, Shaanxi 710049, China \\ Correspondence should be addressed to Aimin Zhang; zhangam@mail.xjtu.edu.cn
}

Received 5 March 2015; Revised 15 April 2015; Accepted 15 April 2015

Academic Editor: Xiaosong Hu

Copyright (c) 2015 Chao Zhang et al. This is an open access article distributed under the Creative Commons Attribution License, which permits unrestricted use, distribution, and reproduction in any medium, provided the original work is properly cited.

\begin{abstract}
This paper deals with the problems of "explosion of term," uncertain parameter in static synchronous compensator (STATCOM) system with nonlinear time-delay. An improved adaptive controller is proposed to enhance the transient stability of system states and reduce computational complexity of STATCOM control system. In contrast to backstepping control scheme in high order systems, the problem of "explosion of term" is avoided by designing dynamic surface controller. The low pass filter is included to allow a design where the model is not differentiated and thus has prevented the mathematical complexities effectively. In addition, unlike the traditional adaptive control schemes, the certainty equivalence principle is not required for estimating the uncertain parameter by system immersion and manifold invariant (I\&I) adaptive control. A smooth function is added to ensure that the estimation error converges to zero in finite time. The effectiveness of the proposed controller is verified by the simulations. Compared with adaptive backstepping and proportion integration differentiation (PID), the oscillation amplitudes of transient response are reduced by nearly half, and the time of reaching steady state is shortened by at least $11 \%$.
\end{abstract}

\section{Introduction}

As a member of flexible alternate current transmission system (FACTS), static synchronous compensator (STATCOM) is being widely used to compensate the reactive power with fast response time by generating or absorbing reactive power continuously in a power system [1-3]. Particularly in sustainable transportation systems, the STATCOM can also be used, such as energy storage [4], renewables integration [5], synergy between electric vehicles, and power systems [6]. It is an effective way of reducing voltage flicker emissions at the point of common coupling (PCC), removing the external fluctuations, and improving the transient stability of system states. However, as dealing with the real STATCOM control problem, the designers are unavoidably to face the difficulties involving uncertain parameter, nonlinear time delay, and complex mathematical models. The nonlinear time-delay is caused by smoothing the harmonic currents and harmonic voltage by using the digital filtering, calculating mean value of each DC voltage and transmitting to protection control unit.
The time-delay ranges from $0.01 \mathrm{~s}$ to $0.04 \mathrm{~s}$. Furthermore, the damping coefficient is difficult to measure accurately in practice, which can be seen as uncertain parameter. Last but most important, since the mathematical modeling is more complicated, the computing complexity is improved significantly in designing STATCOM controller. Therefore, it is a hotspot to study an advanced, reliable, and low complex nonlinear control scheme for STATCOM.

Recently, adaptive backstepping control has been shown as an effective scheme to design STATCOM controllers while keeping better performance $[7,8]$. This method is a vast of research, which is adapted by some intelligent methods, for example, least-squares estimation [9] and various kalman filtering approaches [10-12]. Some STATCOM controllers are designed by using backstepping technique involving the nonlinear robust control law and a new estimator to estimate the uncertain parameters [13-15]. However, in the real STATCOM system, nonlinear time-delay is an important factor that these adaptive backstepping schemes did not include. Moreover, the adaptive backstepping is 
flawed with two shortcomings. Frist, the strong coupling between state variables and estimation errors existed in constructing the control Lyapunov function (CLF) for estimating the uncertain parameter. If the estimator is fixed, the estimation error will be accumulated and the error of coupling term will in turn be accumulated as running time increases [16]. Second, backstepping technique involves model differentiation in STATCOM control system and thus has suffered the "explosion of term." So the computational processes of nonlinear controller leads to high complexity.

The method named the system immersion and manifold invariant (I\&I) adaptive control is developed in [17]. Since the knowledge of the CLF is not required by this method, the problem of the oscillation of states caused by the coupling between state variables and estimation error can be avoided. It has been proved that transient stability of the system can be guaranteed even if estimators reach the limit of its capacity [14]. Therefore, for nonlinear STATCOM system in adaptive law design, this I\&I adaptive control can be adopted to estimate uncertain parameter by designing a smooth function to offset the estimation error. Moreover, the stability and the convergence of the adaptive law are achieved by requiring the estimation error to converge to zero in the finite time.

To overcome the "explosion of term" caused by adaptive backstepping control, the method called dynamic surface control was developed to simplify the controller design where model differentiation can be avoided in higher systems [18]. The low pass filters are included to allow a design where the model is not differentiated, at the same time, avoiding the high computing complexity caused by the "explosion of term." For a class of pure-feedback nonlinear systems, the problem explosion of complexity has been solved by using the dynamic surface technique in [19]. Moreover, semiglobal uniform ultimate boundedness of all signals is guaranteed in the closed-loop system. However, the dynamic surface control cannot be applied to nonlinear STATCOM systems with uncertain parameter; it also did not consider the problem of nonlinear time delay.

In this paper, an improved adaptive controller is proposed to improve stability of STATCOM system by simultaneously addressing the problems involving "explosion of term," uncertain parameter, and nonlinear time-delay. The low pass filters are included to allow a design where the model is not differentiated based on dynamic surface control, at the same time, avoiding the "explosion of term" caused by model differentiation. Furthermore, based on the I\&I adaptive control, the existing uncertain parameter is estimated in adaptive law design. The designed adaptive law can ensure that the estimation error converges to zero in finite time. Moreover, the nonnegative time-delay function is introduced to overcome the effect of nonlinear time delay and achieve the best possible control performance. It is proved that all the state variables are globally bounded and converge to the equilibrium points by using the proposed controller. The simulation results show that transient stability and the convergence speed of the system state variables are improved effectively by the proposed controller.

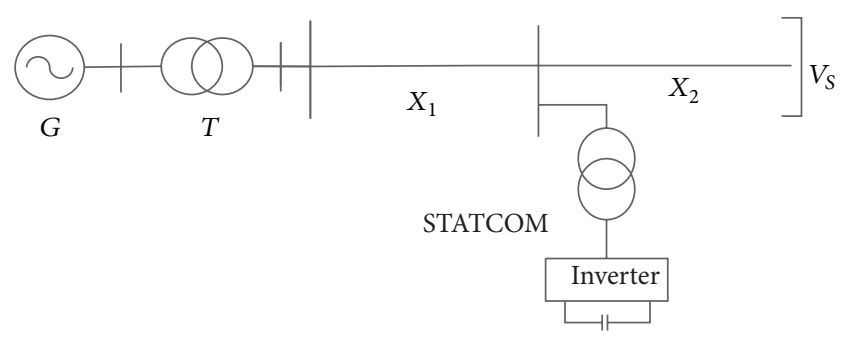

FIGURE 1: STATCOM single machine infinite system model.

\section{System Model and Control Objective}

Consider the single-machine infinite-bus system with STATCOM shown in Figure 1 [20], where $G$ is the alternating current generator and $T$ is the transformer.

It is clearly shown that the STATCOM is installed on the grid, which can instantly and continuously provide variable reactive power in response to voltage transients, supporting the stability of grid voltage.

Its mathematical equivalent system dynamic model can be expressed in (1) by the following nonlinear differential equations:

$$
\begin{aligned}
& \dot{\delta}=\omega-\omega_{0}, \\
& \dot{\omega}=\frac{\omega_{0}}{H}\left[P_{m}-\frac{E_{q}^{\prime} V_{s} \sin \delta}{X_{1}+X_{2}}(1\right. \\
& \left.+\frac{X_{1} X_{2} I_{q}}{\sqrt{\left(X_{2} E_{q}^{\prime}\right)^{2}+\left(X_{1} V_{s}\right)^{2}+2 X_{1} X_{2} E_{q}^{\prime} V_{S} \cos \delta}}\right) \\
& \left.-\frac{D}{H}\left(\omega-\omega_{0}\right)\right] \\
& \dot{I}_{q}=\frac{1}{T_{q}}\left(-I_{q}(t-d)+I_{q 0}+u_{B}\right) \text {, }
\end{aligned}
$$

where $d$ is delay time and the three state variables are generator rotor angle $\delta$, generator rotor angular speed $\omega$, and reactive current $I_{q}$ where transient responses will be tracked. It is noted that the vector $\left[\delta_{0}, \omega_{0}, I_{q 0}\right]^{T}$ is the steady-state operating point. This implies that the steady operation point is the desired value or objective value $[20,21]$. The parameters in (1) are expressed in Appendix A.

To simplify model (1), three state variables are redefined as $x_{1}=\delta-\delta_{0}, x_{2}=\omega-\omega_{0}$, and $x_{3}=I_{q}-I_{q 0}$. The model (1) can be rewritten as

$$
\begin{aligned}
& \dot{x}_{1}=x_{2}, \\
& \dot{x}_{2}=\theta x_{2}+k_{1} P_{m}
\end{aligned}
$$




$$
\begin{aligned}
& -k_{2} \sin \left(\delta_{0}+x_{1}\right)\left[1+f\left(x_{1}\right)\left(x_{3}+I_{q 0}\right)\right], \\
\dot{x}_{3}= & k_{3}\left(-x_{3}(t-d)+u_{B}\right),
\end{aligned}
$$

where

$$
\begin{aligned}
& f\left(x_{1}\right) \\
& =\frac{X_{1} X_{2}}{\sqrt{\left(X_{2} E_{q}^{\prime}\right)^{2}+\left(X_{1} V_{S}\right)^{2}+2 X_{1} X_{2} E_{q}^{\prime} V_{S} \cos \left(x_{1}+\delta_{0}\right)}}, \\
& k_{1}=\frac{\omega_{0}}{H}, \\
& k_{2}=\frac{\omega_{0} E_{q}^{\prime} V_{S}}{H\left(X_{1}+X_{2}\right)}, \\
& k_{3}=\frac{1}{T_{q}} .
\end{aligned}
$$

The damping coefficient $D$ cannot be measured accurately in STATCOM system and the inertia $H$ is a constant. Therefore, the expression $\theta=-D / H$ is also the uncertain parameter.

The objective of designing STATCOM controller is to guarantee that all the state variables are globally bounded and converge to the desired points. This implies that generator rotor angle, generator rotor angular speed, and reactive current of the STATCOM can be adjusted to the equilibriums in the finite time.

\section{Design of STATCOM Controller}

Three are three sections to introduce our proposed controller in designing robust controller. In Section 3.1, I\&I adaptive control is adopted for designing adaptive law. In Section 3.2, dynamic surface control is used for designing control law. In Section 3.3, the stability of STATCOM control system is verified.

3.1. Design of the Adaptive Law. The method I\&I adaptive control can be adopted to the estimate uncertain parameter with the adaptive law design. By adopting this method, the uncertain parameter is estimated in the following steps.

Define a manifold as

$$
e_{\theta}=\hat{\theta}-\theta+\beta\left(x_{1}, x_{2}\right),
$$

where $\theta$ is the uncertain parameter, $\widehat{\theta}$ is the estimation value of $\theta$, and $\beta\left(x_{1}, x_{2}\right)$ is the smooth function to be designed. The derivative of (4) is

$$
\begin{aligned}
\dot{e}_{\theta} & =\dot{\hat{\theta}}+\sum_{k=1}^{2} \frac{\partial \beta}{\partial x_{k}} \times \dot{x}_{k}=\dot{\hat{\theta}}+\frac{\partial \beta}{\partial x_{1}} x_{2}+\frac{\partial \beta}{\partial x_{2}}\left(\theta x_{2}+k_{1} P_{m}\right. \\
& \left.-k_{2} \sin \left(\delta_{0}+x_{1}\right)\left(1+f\left(x_{1}\right)\left(x_{3}+I_{q 0}\right)\right)\right) .
\end{aligned}
$$

In order to cancel the parameter-independent terms, $\dot{\hat{\theta}}$ is designed as

$$
\begin{aligned}
\dot{\hat{\theta}}=- & \frac{\partial \beta}{\partial x_{1}} x_{2}-\frac{\partial \beta}{\partial x_{2}}\left((\widehat{\theta}+\beta) x_{2}+k_{1} P_{m}\right. \\
& \left.\cdot-k_{2} \sin \left(\delta_{0}+x_{1}\right)\left(1+f\left(x_{1}\right)\left(x_{3}+I_{q 0}\right)\right)\right) .
\end{aligned}
$$

Substituting (6) into (5), (5) can be rewritten as

$$
\dot{e}_{\theta}=-\frac{\partial \beta}{\partial x_{2}} e_{\theta} x_{2}
$$

Lemma 1. Define a candidate Lyapunov function (CLF)

$$
V\left(e_{\theta}\right)=\frac{1}{2} e_{\theta}^{2} .
$$

By selecting the smooth function $\beta\left(x_{1}, x_{2}\right)$, we have $\lim _{t \rightarrow \infty} e_{\theta}(t)=0$.

Proof. Theoretically, we have large flexibility in selecting $\beta\left(x_{1}, x_{2}\right)$. For simplicity, we let $\beta\left(x_{1}, x_{2}\right)=(1 / 2) \rho x_{2}{ }^{2}$ with $\rho>0$.

$$
\dot{V}\left(e_{\theta}\right)=e_{\theta} \dot{e}_{\theta}=-\frac{\partial \beta}{\partial x_{2}} e_{\theta}^{2} x_{2}=-e_{\theta}^{2} x_{2}^{2} \leq 0 .
$$

Since the derivative of the CLF (8) is negative semidefinite, the manifold $e_{\theta}$ can converge to zero in finite time based on Lyapunov theorem. As a result, we have $\lim _{t \rightarrow \infty} e_{\theta}(t)=0$ Lemma 1 holds.

Remark 2. By using the designed smooth function, the manifold $e_{\theta}$ can converge to zero in finite time; that is $\lim _{t \rightarrow \infty} e_{\theta}(t)=0$, based on Lyapunov stability theorem. Therefore, based on the theory of immersion and manifold invariant (I\&I) adaptive control, the manifold $e_{\theta}(t)=\widehat{\theta}-$ $\theta+\beta\left(x_{1}, x_{2}\right)=0$ is invariant when $\lim _{t \rightarrow \infty} e_{\theta}(t)=0$, and thus the parametric form manifold $I_{e}=\left\{(x, \widehat{\theta}) \in R^{3} \times R^{1}\right.$ । $\left.\widehat{\theta}-\theta+\beta\left(x_{1}, x_{2}\right)=0\right\}$ is invariant and attractive $[9,10]$.

3.2. Design of the Control Law. Based on dynamic surface control, we can design control law $\left(u_{B}\right)$ in three steps.

Step 1. Error variables $z_{i}(i=1,2,3)$ can be defined as the following:

$$
\begin{aligned}
& z_{1}=x_{1}, \\
& z_{2}=x_{2}-x_{2}{ }^{*}, \\
& z_{3}=x_{3}-x_{3}{ }^{*},
\end{aligned}
$$


where $x_{1}, x_{2}$, and $x_{3}$ are the virtual controls and $x_{2}{ }^{*}$ and $x_{3}{ }^{*}$ are the stabilizing functions. The derivative of error variables $z_{i}(i=1,2,3)$ with $(2)$ is

$$
\begin{aligned}
\dot{z}_{1}= & x_{2}, \\
\dot{z}_{2}= & \dot{x}_{2}-\dot{x}_{2}{ }^{*} \\
= & \theta x_{2}+k_{1} P_{m} \\
& \quad-k_{2} \sin \left(\delta_{0}+x_{1}\right)\left(1+f\left(x_{1}\right)\left(x_{3}+I_{q 0}\right)\right)-\dot{x}_{2}{ }^{*}, \\
\dot{z}_{3}= & \dot{x}_{3}-\dot{x}_{3}{ }^{*}=k_{3}\left(-x_{3}(t-d)+u_{B}\right)-\dot{x}_{3}{ }^{*} .
\end{aligned}
$$

Choose the first CLF as

$$
V_{1}=\frac{1}{2} z_{1}^{2}
$$

The derivative of $V_{1}$ along with (12) is

$$
\dot{V}_{1}=z_{1} z_{2}+z_{1} x_{2}^{*} \text {. }
$$

Take the stabilizing function $x_{2}{ }^{*}$ as

$$
x_{2}^{*}=-c_{1} x_{1}
$$

where $c_{1}$ is a nonnegative constant. It can be seen clearly that $\dot{V}_{1} \leq 0$ if $z_{2}=0$.

Step 2. The second energy storage function with respect to Lyapunov function is

$$
V_{2}=\frac{1}{2} z_{1}^{2}+\frac{1}{2} z_{2}^{2}
$$

The derivative of $V_{2}$ is

$$
\dot{V}_{2}=z_{1} \dot{z}_{1}+z_{2} \dot{z}_{2}
$$

Substituting (11) and (14) into (16), the equation above is manipulated as

$$
\begin{aligned}
\dot{V}_{2} & =z_{1} \dot{z}_{1}+z_{2} \dot{z}_{2}=z_{1} z_{2}-c_{1} z_{1}{ }^{2}+z_{2}\left(\dot{x}_{2}-\dot{x}_{2}{ }^{*}\right) \\
& =z_{1} z_{2}-c_{1} z_{1}{ }^{2}+z_{2}\left(\dot{x}_{2}+c_{1} z_{2}-c_{1}{ }^{2} z_{1}\right)=\left(1-c_{1}{ }^{2}\right) \\
& \cdot z_{1} z_{2}-c_{1} z_{1}{ }^{2}+z_{2}\left[\theta x_{2}+k_{1} P_{m}\right. \\
& \left.-k_{2} \sin \left(\delta_{0}+x_{1}\right)\left(1+f\left(x_{1}\right)\left(x_{3}+I_{q 0}\right)\right)+c_{1} z_{2}\right] .
\end{aligned}
$$

To guarantee this second-order subsystem satisfying Lyapunov stability, the stabilizing function $x_{3}{ }^{*}$ must make (17) satisfy the inequality that $V_{2} \leq 0$. And then, $x_{3}{ }^{*}$ is

$$
\begin{aligned}
x_{3}{ }^{*} & \\
= & \left(\frac{(\hat{\theta}+\beta) x_{2}+k_{1} P_{m}+\left(1-c_{1}^{2}\right) z_{1}+c_{1} z_{2}+c_{2} z_{2}}{k_{2} f\left(x_{1}\right) \sin \left(\delta_{0}+x_{1}\right)}\right) \\
& -\frac{1}{f\left(x_{1}\right)}-I_{q 0} .
\end{aligned}
$$

Remark 3. It would be a tremendous expansion of terms if derivative of (18) is calculated. The problem of high computational complexity can be caused in the following control law design by using backstepping control. The dynamic surface control can be introduced to design the control law and solve the problem of "explosion of term."

The low pass filter $1 /(\tau s+1)$ is included to design control law without model differentiation, which can avoid the problem of "explosion of term" that has made other methods difficult to implement in practice.

The stabilizing function $x_{3}{ }^{*}$ is the output of low-pass filter, and the $\bar{x}_{3}$ is the input of low-pass filter. The relationship between $x_{3}{ }^{*}$ and $\bar{x}_{3}$ is

$$
\begin{aligned}
\tau\left(x_{3}{ }^{*}\right)^{\prime}+x_{3}{ }^{*} & =\bar{x}_{3}, \\
x_{3}{ }^{*}(0) & =\bar{x}_{3}(0) .
\end{aligned}
$$

From (19), we can obtain $\left(x_{3}{ }^{*}\right)^{\prime}=\left(\bar{x}_{3}-x_{3}{ }^{*}\right) / \tau$. The filtering error can be defined as

$$
y=x_{3}{ }^{*}-\bar{x}_{3} \text {. }
$$

A CLF involving time-delay nonlinearity, error variables, and filtering error is designed as

$$
V_{3}=\frac{1}{2} z_{1}^{2}+\frac{1}{2} z_{2}^{2}+\frac{1}{2} z_{3}^{2}+\frac{1}{2} y^{2}+\int_{t-d}^{t} q(x(\alpha)) d \alpha,
$$

where $q(x(t))$ is a nonnegative function. We have the derivative of (21) being

$$
\begin{aligned}
\dot{V}_{3}= & z_{1} \dot{z}_{1}+z_{2} \dot{z}_{2}+z_{3} \dot{z}_{3}+y \dot{y}+q(x(t)) \\
& -q(x(t-d)) .
\end{aligned}
$$

Substituting $\dot{z}_{3}=k_{3}\left(-x_{3}(t)+u_{B}\right)-\dot{x}_{3}{ }^{*}, \dot{y}=\left(\bar{x}_{3}-x_{3}{ }_{3}\right) / \tau-$ $\dot{\bar{x}}_{3}=-y / \tau+B_{3}$ into (22), we can obtain

$$
\begin{aligned}
\dot{V}_{3} & =z_{1} \dot{z}_{1}+z_{2} \dot{z}_{2}+z_{3} \dot{z}_{3}+y \dot{y}+q(x(t)) \\
& -q(x(t-d))=\left(1-c_{1}^{2}\right) z_{1} z_{2}-c_{1} z_{1}^{2}+z_{2}\left[\theta x_{2}\right. \\
& +k_{1} P_{m}-k_{2} \sin \left(\delta_{0}+x_{1}\right)\left(1+f\left(x_{1}\right)\left(x_{3}+I_{q 0}\right)\right) \\
& \left.+c_{1} z_{2}\right]+z_{3}\left(k_{3}\left(-x_{3}(t-d)+u_{B}\right)-\dot{x}_{3}{ }^{*}\right)+y\left(\frac{-y}{\tau}\right. \\
& \left.+B_{3}\right)+q(x(t))-q(x(t-d)),
\end{aligned}
$$

where $B_{3}=-\dot{\bar{x}}_{3}$. Define $h\left(x_{3}(t-d)\right)=\left|k_{3} x_{3}(t-d)\right|$, where $h\left(x_{3}(t-d)\right)$ is a nonnegative time-delay function which can be compensated in the adaptive nonlinear controller design. The nonnegative function can be defined as $q(x(t))=\left|k_{3} z_{3} x_{3}(t)\right|$, which is a reduced form of satisfying Lyapunov stability, but not the only form.

Based on Cauchy-Schwartz inequality theorem, a relational expression can be obtained

$$
\begin{aligned}
-z_{3} h\left(x_{3}(t-d)\right) & \leq\left|k_{3} z_{3} x_{3}(t-d)\right| \\
& \leq\left|z_{3}\right|\left|k_{3} x_{3}(t-d)\right|
\end{aligned}
$$


By $q(x(t))=\left|k_{3} z_{3} x_{3}(t)\right|$, we can get $q(x(t-d))=$ $\left|k_{3} z_{3} x_{3}(t-d)\right|$. Substituting $q(x(t)), q(x(t-d))$ and (24) into (23), we can obtain

$$
\begin{aligned}
\dot{V}_{3} & =z_{1} \dot{z}_{1}+z_{2} \dot{z}_{2}+z_{3} \dot{z}_{3}+y \dot{y}+q(x(t)) \\
& -q(x(t-d)) \leq\left(1-c_{1}{ }^{2}\right) z_{1} z_{2}-c_{1} z_{1}^{2}+z_{2}\left[\theta x_{2}\right. \\
& +k_{1} P_{m}-k_{2} \sin \left(\delta_{0}+x_{1}\right)\left(1+f\left(x_{1}\right)\left(x_{3}+I_{q 0}\right)\right) \\
& \left.+c_{1} z_{2}\right]+z_{3}\left(k_{3} u_{B}-\dot{x}_{3}{ }^{*}\right)+y\left(\frac{-y}{\tau}+B_{3}\right) \\
& +\left|k_{3} z_{3} x_{3}(t)\right| .
\end{aligned}
$$

The control law is designed as

$$
u_{B}=\frac{1}{k_{3}} \dot{x}_{3}{ }^{*}-\frac{c_{3}}{k_{3}} z_{3}+\lambda\left|x_{3}(t)\right|
$$

where $c_{3}>0$, and $\lambda$ is a sign function, which is defined as $\lambda=-1$ when $z_{3}>0$ and $\lambda=1$ when $z_{3}<0$.

\subsection{Proof of System Stability}

Lemma 4. All of state variables of the closed-loop system are bounded and converge to the equilibrium point, if $V(0) \leq p$, $p>0$.

Proof. Let $V=(1 / 2) z_{1}^{2}+(1 / 2) z_{2}^{2}+(1 / 2) z_{3}^{2}+(1 / 2) y^{2}=p$, $B_{3}$ is bounded, which is denoted as $M_{3}$, and then we have $B_{3}{ }^{2} / M_{3}{ }^{2}-1 \leq 0$. Substituting (18) and (26) to (25), we can obtain

$$
\begin{aligned}
\dot{V} & =z_{1} \dot{z}_{1}+z_{2} \dot{z}_{2}+z_{3} \dot{z}_{3}+y \dot{y} \\
& \leq-c_{1} z_{1}^{2}-c_{2} z_{2}^{2}-z_{2} x_{2} e_{\theta}-c_{3} z_{3}^{2}+y\left(\frac{-y}{\tau}+B_{3}\right) .
\end{aligned}
$$

Based on Cauchy-Schwartz inequality theorem, (27) can be rewritten as follows:

$$
\begin{aligned}
\dot{V}= & z_{1} \dot{z}_{1}+z_{2} \dot{z}_{2}+z_{3} \dot{z}_{3}+y \dot{y} \\
\leq & -c_{1} z_{1}{ }^{2}-c_{2} z_{2}^{2}-z_{2} x_{2} e_{\theta}-c_{2} z_{3}{ }^{2}-\frac{y^{2}}{\tau}+|y|\left|B_{3}\right| \\
\leq & -c_{1} z_{1}{ }^{2}-c_{2} z_{2}{ }^{2}-z_{2} x_{2} e_{\theta}-c_{2} z_{3}{ }^{2}-\frac{y^{2}}{\tau}+\frac{1}{2} B_{3}{ }^{2} y^{2} \\
& +\frac{1}{2} \\
= & -c_{1} z_{1}{ }^{2}-c_{2} z_{2}{ }^{2}-c_{3} z_{3}{ }^{2}-z_{2} x_{2} e_{\theta} \\
& +\left(\frac{1}{2} B_{3}{ }^{2}-\frac{1}{\tau}\right) y^{2}+\frac{1}{2} .
\end{aligned}
$$

By designing $c_{1} \geq r, c_{2} \geq r, c_{3} \geq r$, and $1 / \tau \geq(1 / 2) M_{3}{ }^{2}+r$, $r \geq 0$, we can obtain

$$
\begin{aligned}
\dot{V} \leq & -r z_{1}^{2}-r z_{2}^{2}-r z_{3}^{2}+\left(\frac{1}{2} B_{3}^{2}-\frac{M_{3}^{2}}{2}-r\right) y^{2} \\
& +\frac{1}{2}=-2 r V+\left(\frac{M_{3}}{2 M_{3}} B_{3}^{2}-\frac{M_{3}^{2}}{2}\right) y^{2}+\frac{1}{2} \\
= & -2 r V+\left(\frac{B_{3}^{2}}{M_{3}}-1\right) \frac{M_{3}^{2} y^{2}}{2}+\frac{1}{2} .
\end{aligned}
$$

Substituting $r \geq 1 / 4 p$ into (29), (29) can be rewritten as

$$
\dot{V} \leq-2 \frac{1}{4 p} p+\frac{1}{2}=0 .
$$

From (30), we have $V(t) \leq p$, if $V(0) \leq p$, where $t \geq 0$. Lemma 4 holds.

In addition, the convergence analysis is also given. From (29), we can get

$$
\dot{V} \leq-2 r V+\frac{1}{2} .
$$

Solve this differential equation as

$$
V \leq \frac{1}{4 r}+\left(V(0)-\frac{1}{4 r}\right) e^{-2 r t} .
$$

If $t \rightarrow \infty, V \rightarrow 1 / 4 r$, and then we have $V \rightarrow 1 / 4 r$, when $r \rightarrow \infty$. Furthermore, due to $1 / \tau \geq(1 / 2) M_{3}{ }^{2}+1 / 2+r$, we can obtain $r \rightarrow \infty$, when $\tau \rightarrow 0$. It is an important basis for design of low pass filter $1 /(\tau s+1)$.

\section{Simulation Results and Discussion}

In this section, the simulation model of adaptive nonlinear controller has been established under the MATLAB/Simulink environment for nonlinear STATCOM with nonlinear timedelay. The parameters in (1) are given as follows.

Consider $H=8 \mathrm{~s}, E_{q}^{\prime}=1.108 \mathrm{pu}, P_{m}=1.0 \mathrm{kw}, V_{s}=1 \mathrm{pu}$, $X_{1}=0.84 \mathrm{pu}, X_{2}=0.52 \mathrm{pu}, T_{q}=0.03 \mathrm{~s}, p=2, c_{1}=1, c_{2}=1$, $c_{3}=1$, and $d=0.02 \mathrm{~s}, 0.04 \mathrm{~s}$. The steady operation points are given as $\delta_{0}=57.1^{\circ}, \omega_{0}=314.159 \mathrm{rad} / \mathrm{s}, I_{0}=0$, and $y(0)=0$.

The transient responses of the nonlinear STATCOM system with time-delay are then discussed. A comparison analysis with the conventional nonlinear controller is also provided under the same conditions.

(1) Different Control Approaches. The comparison between the proposed dynamic surface method for nonlinear STATCOM with time-delay based on system immersion and manifold invariant methodology (DSMII) approach and two approaches involving adaptive backstepping (AB) [21] and proportion integration differentiation (PID) [22] were investigated, when $d=0.02 \mathrm{~s}$.

Figures 2(a)-2(c) show the comparison between the proposed controller and the two controllers when $d=0.02 \mathrm{~s}$. 


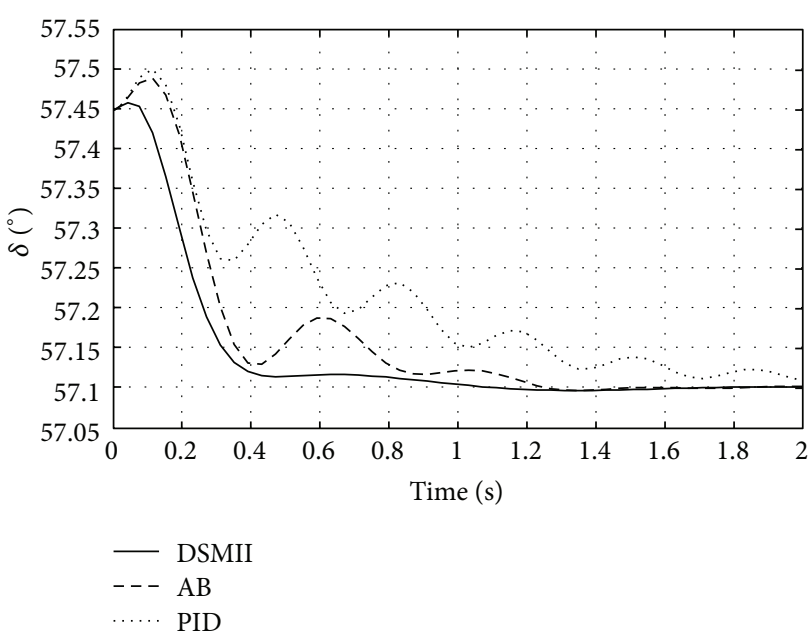

(a)

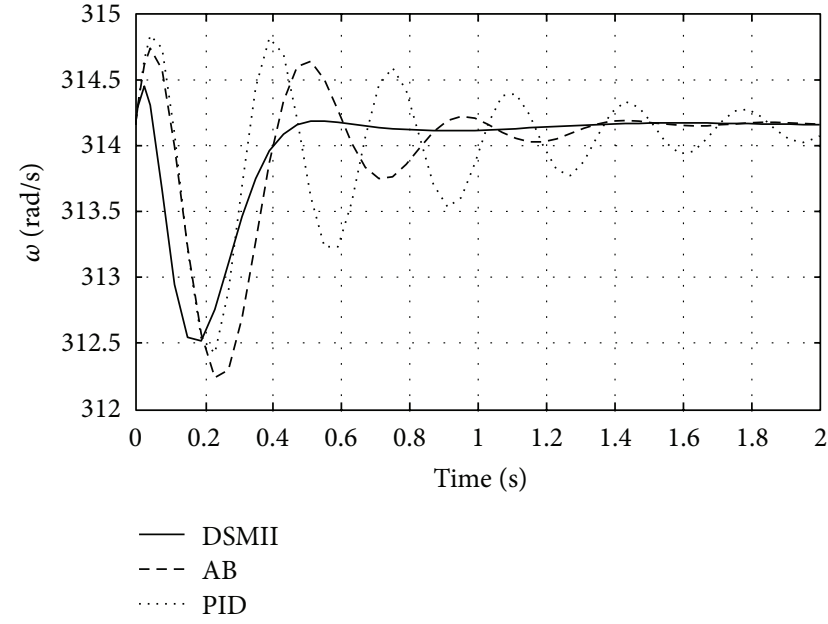

(b)

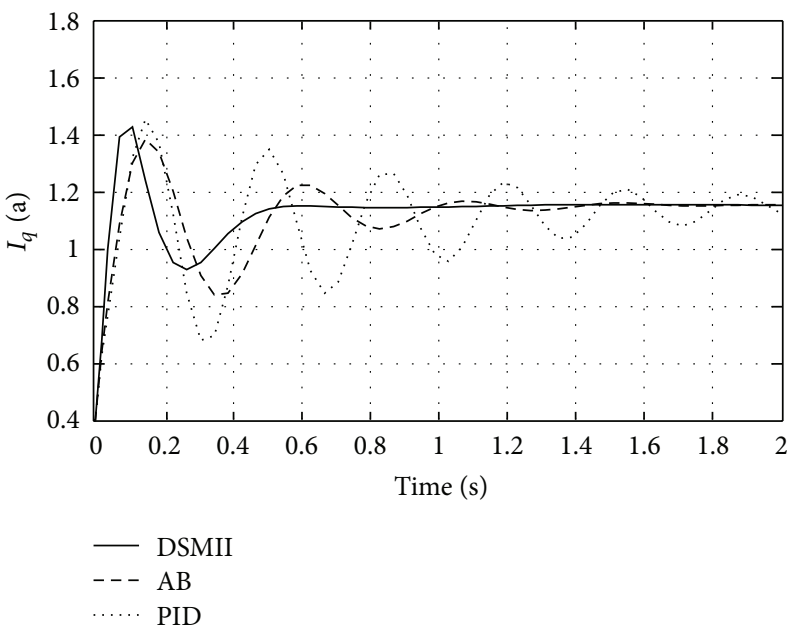

(c)

Figure 2: (a) Transient responses of rotor angle when $d=0.02 \mathrm{~s}$. (b) Transient responses of rotor angular speed when $d=0.02 \mathrm{~s}$. (c) Transient responses of reactive current when $d=0.02 \mathrm{~s}$.

For the proposed controller, it can be clearly seen that the convergences of transient responses trajectories are achieved and the system tend to be stable state more rapidly after a very short time. Taking Figure 2(c) for example, the transient responses fluctuate fast and tend to be stable after $1.6 \mathrm{~s}$ or more under $\mathrm{AB}$ and PID. Instead, by using the proposed controller, transient responses fluctuate more smoothly and converge to stable state after $0.6 \mathrm{~s}$, suggesting that the proposed controller results in better system performance.

(2) Different Time Delay. Simulations of our proposed controller are performed at $d_{1}=0.02$ and $d_{1}=0.04$, respectively.

In Figures 3(a)-3(c), we simulated the model in two different delay times to investigate its influences. The transient trajectories depart from the initial state and fluctuate strongly without an appropriate control. From the comparison between Figures 2(a)-2(c) and Figures 3(a)-3(c), all transient trajectories fluctuate faster, and system reaches the stable state more quickly when $d=0.02 \mathrm{~s}$. Moreover, more time is spent for the transient responses to converge to the stable state when $d=0.04 \mathrm{~s}$. It is noted that the transient trajectories fluctuate powerfully and cannot reach steady state in finite time under $\mathrm{AB}$. Consequently, the delay time $d$ is a crucial nonlinear factor impacting the transient and steady performance of the STATCOM system. A larger $d$ can result in a poorer robustness and worse convergence. This result is consistent with the theoretical analysis.

\section{Conclusions}

This paper presents an improved adaptive controller to address the problems of "explosion of term" and uncertain parameter in static synchronous compensator (STATCOM) with nonlinear time delay. Improvements are achieved in three aspects as follows.

(1) The uncertain parameter is estimated by I\&I adaptive control in designing adaptive law, which can ensure 


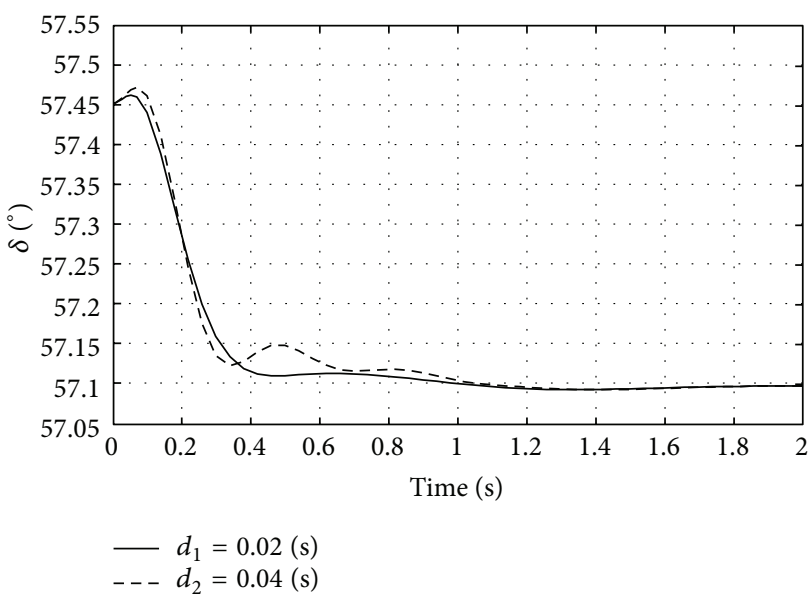

(a)

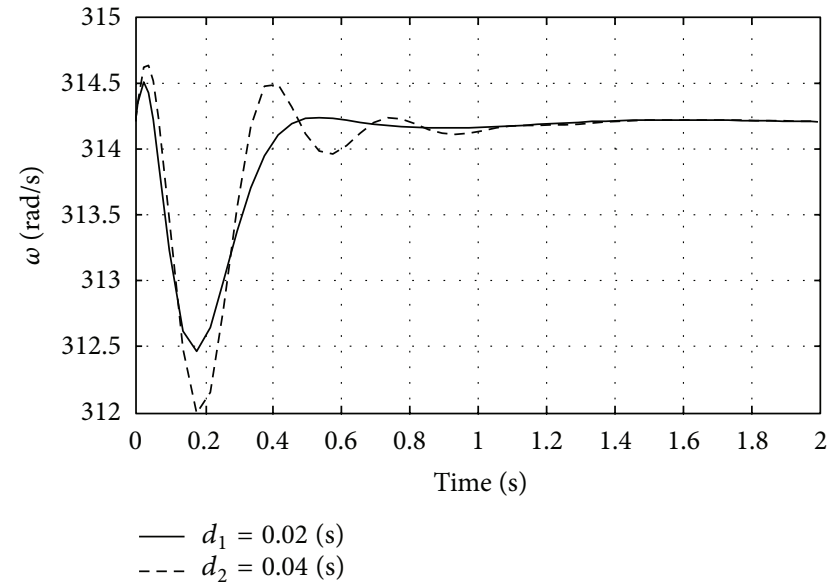

(b)

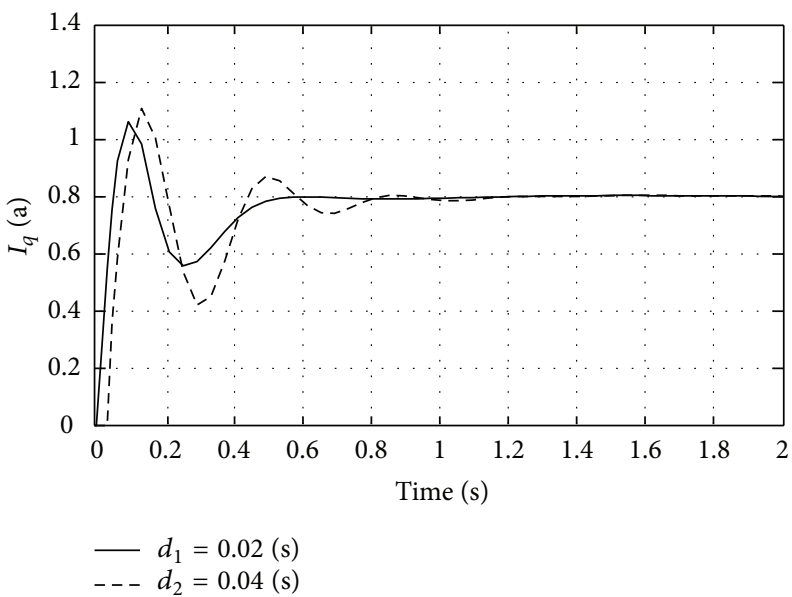

(c)

Figure 3: (a) Transient responses of rotor angle in different time delay. (b) Transient responses of rotor angular speed in different time delay. (c) Transient responses of reactive current in different time delay.

that the estimation error converges to zero in finite time.

(2) With regards to "explosion of term" caused by backstepping technology, a low pass filter is included to allow a design where the model is not differentiated by using dynamic surface control.

(3) Furthermore, the proposed method can add a nonnegative time-delay function to compensate the timedelay term, which can avoid the influence of timedelay term and achieve the best possible control performance.

By comparing with some conventional controller, the proposed controller has advantages in terms of enhancing transient stability and reducing computational complexity. Simulations results show that the proposed controller not only is insensitive to time-delay term but also reduces the convergence time and oscillation amplitude.

\section{Appendices}

\section{A. Nomenclature}

$\delta$ : Generator rotor angle

$\omega$ : Generator rotor angular speed

$I_{q}$ : Reactive current

$d$ : Time delay

$c$ : Adjustable parameter

$y$ : Output

$H$ : Inertia constant

$E_{q}^{\prime}$ : Transient electromotive force

$P_{m}$ : Mechanical power

$D$ : Damping coefficient

$X_{1,2}$ : Equivalent impedance

$u_{B}$ : Equivalence input 
$T_{q}:$ Time constant

$V_{s}$ : Infinite bus voltage.

\section{B. AB and PID Controller}

The $\mathrm{AB}$ controller with the control law is

$$
\begin{aligned}
u_{B} & =x_{3}+T\left\{\left[\frac{1}{n_{1}} m_{1} x_{2}+\left(m_{2}+\hat{\theta}\right)\left(\theta \widehat{x}_{2}+k_{1} P_{m}\right.\right.\right. \\
& \left.\left.-n_{1}\left(1+f\left(x_{1}\right)\left(x_{3}+I_{q 0}\right)\right)\right)+\dot{\hat{\theta}} x_{2}\right] \\
& -\frac{1}{n_{1}^{2}}\left[x_{2} n_{2}\left(m_{1} x_{1}+m_{2} x_{2}+\widehat{\theta} x_{2}+k_{1} P_{m}\right)\right] \\
& \cdot \frac{1}{f\left(x_{1}\right)}+\frac{n_{1} k_{3} f\left(x_{1}\right) x_{2}}{k_{1}}\left[\frac { 1 } { n _ { 1 } } \left(m_{1} x_{1}+m_{2} x_{2}\right.\right. \\
& \left.\left.+\widehat{\theta} x_{2}+k_{1} P_{m}\right)-1\right]-\left[\frac{\left(m_{2}+\widehat{\theta}\right)}{n_{1} f\left(x_{1}\right) \gamma}\right]^{2} e^{3}-\frac{e^{3}}{\gamma^{2}} \\
& \left.-\mu\left|k_{3} x_{3}(t)\right|\right\} .
\end{aligned}
$$

The parameters in simulations are given as follows.

$H=8 \mathrm{~s}, E_{q}^{\prime}=1.108 \mathrm{pu}, T_{q}=0.03 \mathrm{~s}, c_{1}=2, \gamma=0.2, \rho=2$, $q_{1}=0.4, q_{2}=0.6, \sigma=1, E_{q}^{\prime}=1.108 \mathrm{pu}, V_{s}=1, X_{1}=0.84 \mathrm{pu}$, $X_{2}=0.52 \mathrm{pu}, \delta_{0}=57.1^{\circ}, \omega_{0}=314.159 \mathrm{rad} / \mathrm{s}$, and $I_{0}=0$.

The PID controller with the control law is

$$
\begin{aligned}
u_{B}= & R(X)+\frac{H X_{\Sigma}^{2} T_{q}}{E_{q}^{\prime} V_{s} X_{1} X_{2} \sin \delta} \\
& \cdot\left(-k_{l} \int \Delta \omega^{\prime} d t-k_{p} \Delta \omega^{\prime}-\frac{k_{d} d\left(\Delta \omega^{\prime}\right)}{d t}\right),
\end{aligned}
$$

where

$$
\begin{aligned}
R(X)= & \frac{X_{\Sigma}{ }^{2} T_{q}}{E_{q}^{\prime} V_{s} X_{1} X_{2} \sin \delta} \dot{P}_{m}-\frac{X_{\Sigma}{ }^{2} T_{q} D}{E_{q}^{\prime} V_{s} X_{1} X_{2} \sin \delta} \Delta \dot{\omega} \\
& -\frac{X_{\Sigma} T_{q} \operatorname{con} \delta}{X_{1} X_{2} \sin \delta} \Delta \omega-I_{q}+I_{q 0} .
\end{aligned}
$$

The PID parameter is set as

$$
\left[k_{d}, k_{p}, k_{l}\right]^{T}=[1.3,3.15,4.2]^{T} .
$$

The parameters in simulations are given as follows: $H=$ $8 \mathrm{~s}, E_{q}^{\prime}=1.108 \mathrm{pu}, T_{q}=0.03 \mathrm{~s} P_{m}=1.0 \mathrm{kw}, V_{s}=1 \mathrm{pu}, X_{1}=$ $0.84 \mathrm{pu}, X_{2}=0.52 \mathrm{pu}, \delta_{0}=57.1^{\circ}, \omega_{0}=314.159 \mathrm{rad} / \mathrm{s}$, and $I_{0}=0$.

\section{Conflict of Interests}

The authors declare that there is no conflict of interests regarding the publication of this paper.

\section{Acknowledgments}

This work was supported by National Natural Science Foundation of China (51177126, 61105126), Major Technological Innovation Project Special Fund of Shaanxi Province (2008ZKC01-09), and Applied Fundamental Research Project (SYG201201).

\section{References}

[1] P. Rao, M. L. Crow, and Z. Yang, "STATCOM control for power system voltage control applications," IEEE Transactions on Power Delivery, vol. 15, no. 4, pp. 1311-1317, 2000.

[2] A. Astolfi, D. Karagiannis, and R. Ortega, Nonlinear and Adaptive Control with Applications, Springer Science \& Business Media, 2007.

[3] W. Song and A. Q. Huang, "Fault-tolerant design and control strategy for cascaded H-bridge multilevel converter-based STATCOM," IEEE Transactions on Industrial Electronics, vol. 57, no. 8, pp. 2700-2708, 2010.

[4] X. Hu, S. Li, H. Peng, and F. Sun, "Charging time and loss optimization for LiNMC and $\mathrm{LiFePO}_{4}$ batteries based on equivalent circuit models," Journal of Power Sources, vol. 239, pp. 449-457, 2013.

[5] X. S. Hu, N. Murgovski, L. M. Johannesson, and B. Egardt, "Comparison of three electrochemical energy buffers applied to a hybrid bus powertrain with simultaneous optimal sizing and energy management," IEEE Intelligent Transportation Systems Magazine, vol. 15, no. 3, pp. 1193-1205, 2014.

[6] L. Zhang, Z. Wang, X. Hu, F. Sun, and D. G. Dorrell, "A comparative study of equivalent circuit models of ultracapacitors for electric vehicles," Journal of Power Sources, vol. 274, pp. 899906, 2015.

[7] M. A. Mohd Basri, A. R. Husain, and K. A. Danapalasingam, "Intelligent adaptive backstepping control for MIMO uncertain non-linear quadrotor helicopter systems," Transactions of the Institute of Measurement and Control, vol. 37, no. 3, pp. 345-361, 2015.

[8] J. Zhou, C. Wen, and Y. Zhang, "Adaptive backstepping control of a class of uncertain nonlinear systems with unknown backlash-like hysteresis," IEEE Transactions on Automatic Control, vol. 49, no. 10, pp. 1751-1757, 2004.

[9] X.-S. Hu, F.-C. Sun, and Y. Zou, "Online model identification of lithium-ion battery for electric vehicles," Journal of Central South University of Technology, vol. 18, no. 5, pp. 1525-1531, 2011.

[10] X. S. Hu, F. Sun, and Y. Zou, "Comparison between two modelbased algorithms for Li-ion battery SOC estimation in electric vehicles," Simulation Modelling Practice and Theory, vol. 34, pp. 1-11, 2013.

[11] F. Sun, X. Hu, Y. Zou, and S. Li, "Adaptive unscented Kalman filtering for state of charge estimation of a lithium-ion battery for electric vehicles," Energy, vol. 36, no. 5, pp. 3531-3540, 2011.

[12] L. Zhang, Z. Wang, F. Sun, and D. G. Dorrell, "Online parameter identification of ultracapacitor models using the extended Kalman filter," Energies, vol. 7, no. 5, pp. 3204-3217, 2014.

[13] Y. Zhang, C. Wen, and Y. C. Soh, "Adaptive backstepping control design for systems with unknown high-frequency gain," IEEE Transactions on Automatic Control, vol. 45, no. 12, pp. 23502354, 2000

[14] H. Farokhi Moghadam and N. Vasegh, "Robust PID stabilization of linear neutral time-delay systems," International Journal 
of Computers Communications \& Control, vol. 9, no. 2, pp. 201208, 2014.

[15] L. Zhang, A. Zhang, Z. Ren, G. Li, C. Zhang, and J. Han, "Hybrid adaptive robust control of static var compensator in power systems," International Journal of Robust and Nonlinear Control, vol. 24, no. 12, pp. 1707-1723, 2014.

[16] D. Karagiannis and A. Astolfi, "Nonlinear adaptive control of systems in feedback form: an alternative to adaptive backstepping," Systems \& Control Letters, vol. 57, no. 9, pp. 733-739, 2008.

[17] A. Astolfi and R. Ortega, "Immersion and invariance: a new tool for stabilization and adaptive control of nonlinear systems," IEEE Transactions on Automatic Control, vol. 48, no. 4, pp. 590606, 2003.

[18] D. Swaroop, J. K. Hedrick, P. P. Yip, and J. C. Gerdes, "Dynamic surface control for a class of nonlinear systems," IEEE Transactions on Automatic Control, vol. 45, no. 10, pp. 1893-1899, 2000.

[19] S. J. Yoo, J. B. Park, and Y. H. Choi, "Adaptive dynamic surface control for stabilization of parametric strict-feedback nonlinear systems with unknown time delays," IEEE Transactions on Automatic Control, vol. 52, no. 12, pp. 2360-2364, 2007.

[20] N. Jiang, S. Li, T. Liu, and X. Dong, "Nonlinear large disturbance attenuation controller design for the power systems with STATCOM," Applied Mathematics and Computation, vol. 219, no. 20, pp. 10378-10386, 2013.

[21] W. L. Li, Y. W. Jing, X. P. Liu, and B. Wang, "Nonlinear robust control based on adaptive backstepping design for STATCOM," Journal of Northeastern University (Natural Science), vol. 24, pp. 221-224, 2003.

[22] C. Zhang, Z. Aimin, Z. Hang et al., "An advanced adaptive backstepping control method for STATCOM," in Proceedings of the 26th Chinese Control And Decision Conference (CCDC '14), pp. 1822-1827, IEEE, Changsha, China, May 2014. 


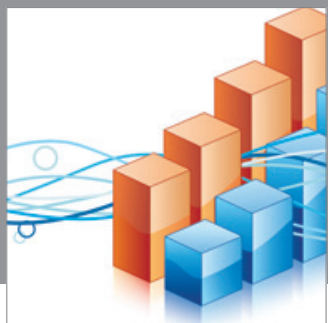

Advances in

Operations Research

mansans

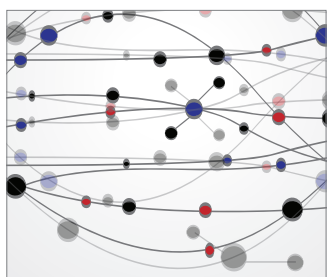

The Scientific World Journal
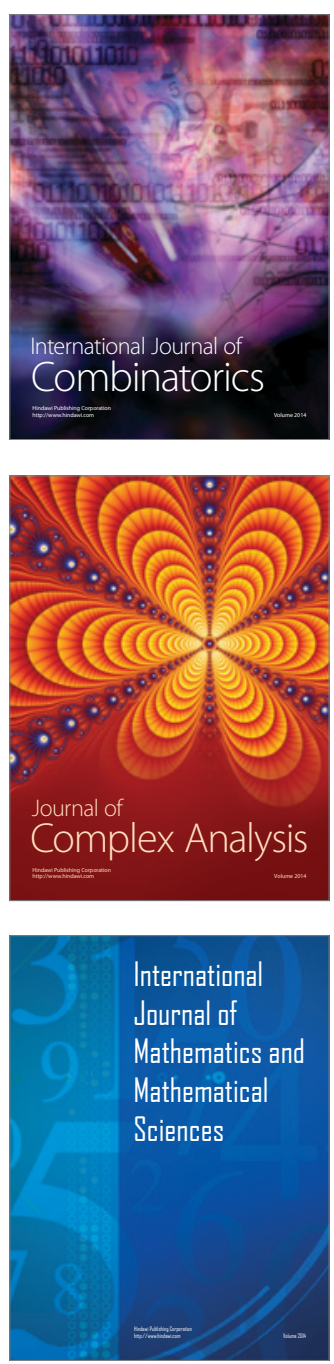
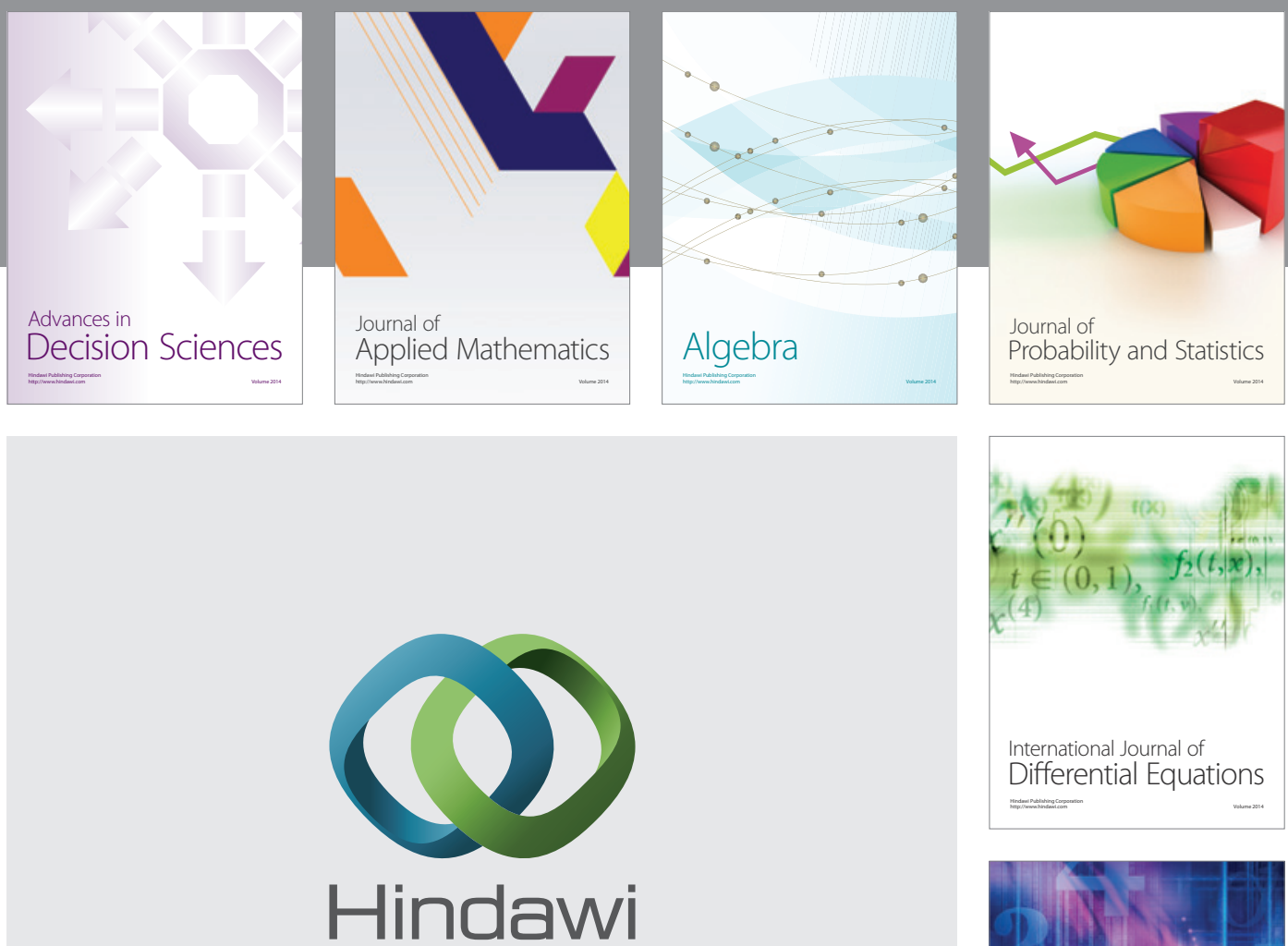

Submit your manuscripts at http://www.hindawi.com
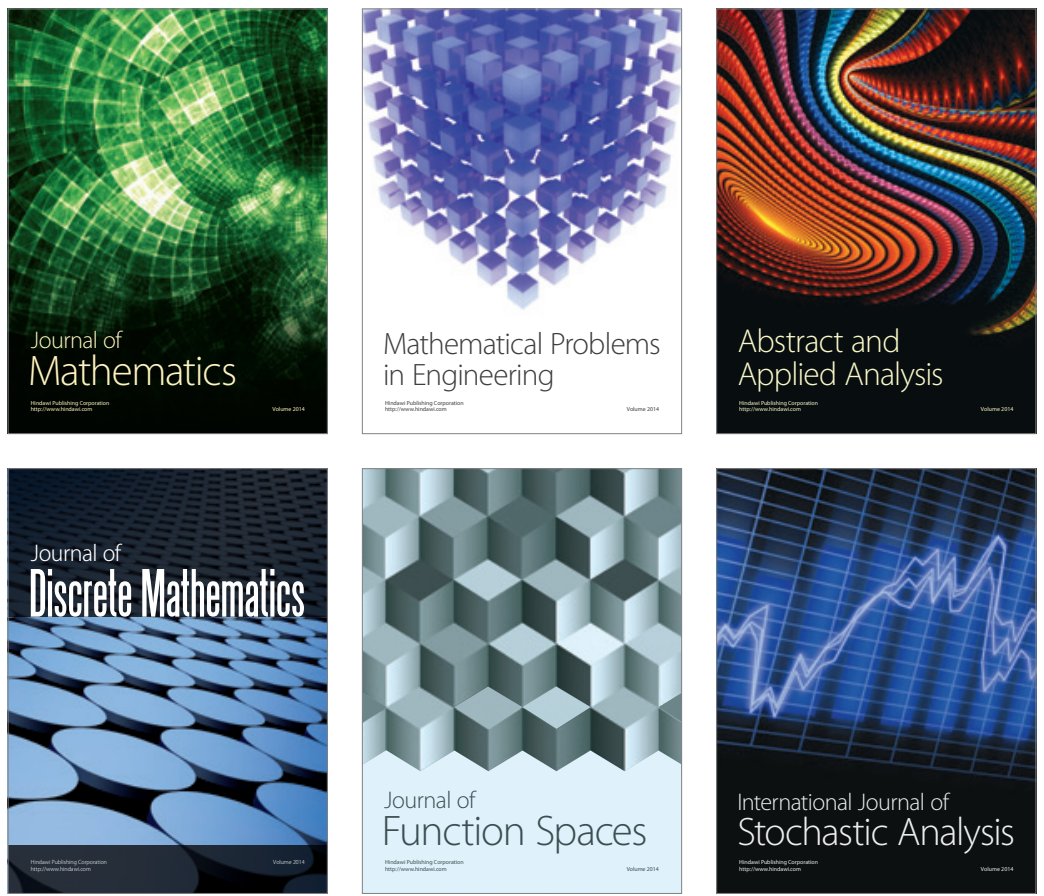

Journal of

Function Spaces

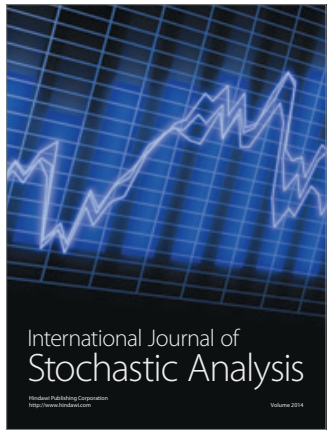

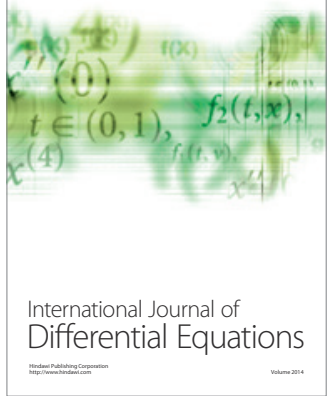
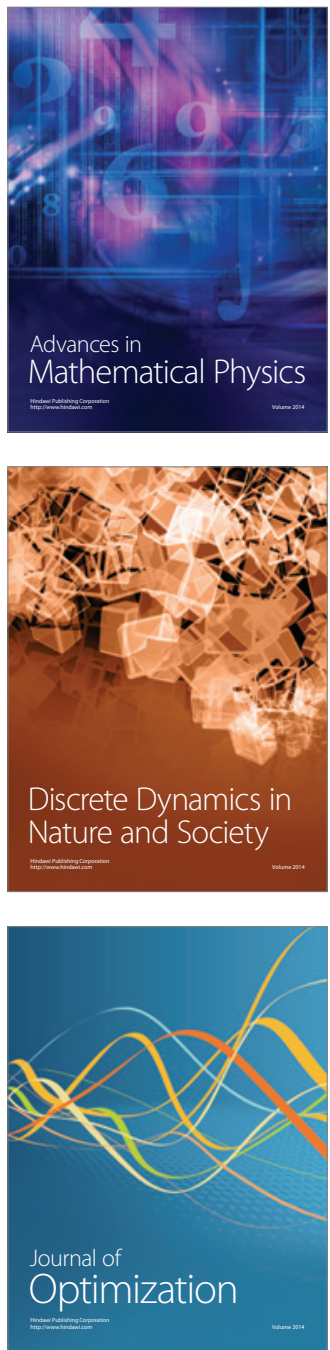